\title{
ACEIs, ARBs, Ibuprofen linked to COVID-19: The other side of the broken mirror
}

\author{
Mina Kelleni ${ }^{1}$ \\ ${ }^{1}$ Affiliation not available
}

June 2, 2020

\begin{abstract}
This article discusses from a pharmacological point of view, the points of weakness in a correspondence published at the Lancet Respiratory Medicine that has suggested a hypothesis relating ACEIs, ARBs and ibuprofen to be associated with a higher risk for COVID-19 infection and complications. This article also explains some unfortunate mistakes that have been made by some who have adopted this hypothesis and decided to unwisely recommend against these important drugs.
\end{abstract}

ACEIs, ARBs, Ibuprofen linked to COVID-19: The other side of the broken mirror

Mina T. Kelleni, MD, PhD

Pharmacology department, College of Medicine, Minia University, Egypt

Pharmacology department, College of Pharmacy, Jouf University, KSA

Email: drthabetpharm@yahoo.com,mina.kelleni@mu.edu.eg, mtkelleni@ju.edu.sa

Mobile: +966560407874

Abstract:

This article discusses from a pharmacological point of view, the points of weakness in a correspondence published at the Lancet Respiratory Medicine that has suggested a hypothesis relating ACEIs, ARBs and ibuprofen to be associated with a higher risk for COVID-19 infection and complications. This article also explains some unfortunate mistakes that have been made by some who have adopted this hypothesis and decided to unwisely recommend against these important drugs.

Key words: SARS CoV-2, COVID-19, ACEIs, ARBs, Ibuprofen

Abbreviations: ACEIs; angiotensin converting enzyme inhibitors, ARBs; angiotensin receptor blockers, SARS-CoV-2; severe acute respiratory syndrome coronavirus 2, COVID-19; Coronavirus disease 2019.

Several reports have indicated that hypertension, diabetes and coronary heart disease are more frequent to be encountered among COVID-19 patients and to be more associated with its complications ${ }^{1-3}$. Many researchers have tried to find a possible relationship and some have hypothesized that "patients treated with ACE2-increasing drugs like ACEIs and ARBs, Ibuprofen and thiazolidinediones are at higher risk for severe COVID-19 infection and, therefore, should be monitored for ACE2-modulating medications" 4. This hypothesis has subsequently led to a medical controversy regarding ACEIs and ARBs as well as to a recommendation against the less vital and much easier to be unwisely replaced; ibuprofen to be administered to COVID-19 patients as declared by France's minister of solidarity and health and initially by the World Health Organization before rapidly changing its stance to declare clearly that it doesn't recommend against 
ibuprofen for COVID-19 patients ${ }^{5}{ }^{6}$. The main aim of this review is to examine the points of weakness of this hypothesis from a pharmacological point of view.

It has been demonstrated that SARS-CoV-2 causing COVID-19 engages the same receptor ACE2 of its predecessor SARS-CoV as the entry receptor to invade its host cells ${ }^{78}$. Moreover, it was suggested that that SARS-CoV-2 has a more efficient binding affinity for ACE2 receptor, which regulates both the cross-species and human-to-human transmissions, than SARS-CoV reasoning for COVID-19 higher contagion ability ${ }^{9}$. In their correspondence ${ }^{10}$, some colleagues have claimed that the expression of ACE2 is substantially increased in patients with type 1 or type 2 diabetes, who are treated with ACEIs or ARBs and thus implying a possible cause for their higher risk for complications when infected with SARS-CoV-2 and they've cited a reference to support their claim ${ }^{11}$. To the best of the author knowledge, this mentioned reference doesn't support their serious suggestion. Further, our colleagues have claimed that: "hypertension is also treated with ACE inhibitors and ARBs, which results in an upregulation of ACE2" citing another reference ${ }^{12}$ which, again the author suggests it has no strong relationship to their suggestion. On the contrary, it states that the activity and action of ACE2 are not affected by ACEIs and it also contradicts their pervious suggestion stating clearly that ACE2 expression is downregulated in diabetes ${ }^{13}$. Noteworthy, upon their subsequent reply to some authors who have requested caution to be mandatorily practiced when recommending discontinuation or providing alternative treatment of ACEIs and ARBs ${ }^{14}$; our colleagues have cited other references ${ }^{15}$ which are also strongly argued by other researchers ${ }^{16}$.

Moreover, our colleagues have stated that ibuprofen and the group of antidiabetic drugs thiazolidinediones which are mostly obsolete, except in the developing countries ${ }^{17}$ have similar effects on their proposed ACE2 expression citing no references at all. The author must stress, with utmost respect that the editor, and more importantly the peer reviewers of this correspondence, shouldn't have ever published such a serious suggestion without enough scientific support. To the best of the author's pharmacological research, there's a single experimental research that gathered both drugs and showed a protective effect of ibuprofen similar to pioglitazone, a member of the thiazolidinediones, against cardiac fibrosis in a rat model partially through their ability to increase ACE2 but in a protective manner ${ }^{18}$. Our colleagues might have interpreted these protective results found in rats for ibuprofen and thiazolidinediones to be unfortunately presented as a hazardous risk for COVID-19 potential patients. It's worthwhile that one of the researchers who published this hypothesis has officially announced in a statement published by his university after five days only form publishing the correspondence, that their suggestions was formulated as a hypothesis regarding ibuprofen and the other drugs and were intended for researchers not for patients and should not be considered as recommendations against usage of these drugs ${ }^{19}$. Unfortunately, this wasn't mentioned at least in a clear manner in their original correspondence; instead they've suggested an alternative for ACEIs and ARBs. Interestingly, the author has recently published a contradictory theory suggesting ibuprofen to be beneficial for COVID-19 20

Yet, were they right in their hypothesis or not? ACE2 is normally highly expressed in the lungs and heart performing a protective physiological role and this has been shown when SARS-CoV induced ACE2 severe down regulation led to an imbalance between angiotensin 1-7 and angiotensin II levels as well as induction of release many inflammatory cytokines that caused acute myocardial dysfunction and lung failure $\mathrm{e}^{21} 22$. Noteworthy, it's also been suggested by experts in the field that the same effect on ACE2 expression may occur with SARS-CoV-2 ${ }^{7}$. Similarly, ACE2 is normally highly expressed in the kidneys with an overexpression that has been shown early in diabetic patients producing a renal protective function, whereas ACE2 subsequent down regulation or induced loss of expression has exacerbated diabetic kidney injury both clinically and experimentally ${ }^{23-25}$. Further, three major American entities associated with cardiology have issued a joint statement just few days after our colleagues have published their hypothesis to dispel the misinformation circulating about the use of ACEIs and ARB medications among patients with COVID-19, recommending their continuation to all patients ${ }^{26}$. Similarly, the European medicines agency has adopted the same conclusion urging the patients not to interrupt their treatment with ACEIs or ARBs as well as not to switch to other medicines stating clearly that there's currently no evidence from clinical or epidemiological studies that establishes a link between ACE inhibitors or ARBs and the worsening of COVID-1927. Interestingly, 
in a study involved 205 acute British inpatients with COVID-19 whom have been adjusted for age, gender as well as comorbidities including diabetes mellitus, hypertension, ischemic heart disease and heart failure; ACEIs have not only been showed to possess adverse effects in those patients, but also they have resulted in a lower rate of death or transfer to a critical care unit within seven days suggesting a potential beneficial effects $^{28}$.

Finally, the author requests the national as well as international health care official authorities to carefully assess their drug recommendations that might affect the medical care, and to avoid recommending against any drug without a proof coming from a well-designed clinical trial. The author would also like to report that he tried starting from the $21^{\text {st }}$ of March, 2020 to publish the main body of this article and faced multiple rejections without peer review from more than 10 top medical journals and I'd like to truly express my utmost respect to the honorable authors, editors, reviewers, journals as well as health care authorities criticized in this review, positive criticism from any researcher no matter how much junior or senior should be always welcomed as our sole concern is the best interests of our precious patients.

Conflict of interests

None

Funding

None

Acknowledgment

The author would like to humbly acknowledge NEJM (three times, one was requested), Nature Medicine, The Lancet, The Lancet Respiratory Medicine, JAMA, Arteriosclerosis, Thrombosis, and Vascular Biology, Antiviral Research, Pharmacological Research, PLOS ONE and Clinical Pharmacology \& Therapeutics for rejecting to publish this manuscript without peer review, and to thank Biomedicine \& Pharmacotherapy for sending the only comment: "The review is premature for publication and should include high quality pathway analysis and emphasis on mechanism of action". Their rejections have sharpened his will and improved his writing skills to potentially improve his current modest 6.5 score in the IELTS.

References:

1. Chen C, Chen C, Yan JT, et al. [Analysis of myocardial injury in patients with COVID-19 and association between concomitant cardiovascular diseases and severity of COVID-19]. Zhonghua Xin Xue Guan Bing Za Zhi 2020;48(0):E008. doi: 10.3760/cma.j.cn112148-20200225-00123 [published Online First: 2020/03/07]

2. Zhang JJ, Dong X, Cao YY, et al. Clinical characteristics of 140 patients infected with SARS-CoV-2 in Wuhan, China. Allergy 2020 doi: 10.1111/all.14238 [published Online First: 2020/02/23]

3. Deng SQ, Peng HJ. Characteristics of and Public Health Responses to the Coronavirus Disease 2019 Outbreak in China. J Clin Med2020;9(2) doi: 10.3390/jcm9020575 [published Online First: 2020/02/26]

4. Fang L, Karakiulakis G, Roth M. Are patients with hypertension and diabetes mellitus at increased risk for COVID-19 infection? The Lancet Respiratory Medicine doi: 10.1016/S2213-2600(20)30116-8

5. The Local. UPDATE - Coronavirus: French health minister and WHO issue warning over taking anti-inflammatories 2020, March 14 [Available from: https://www.thelocal.fr/20200314/coronavirus-frenchhealth-minister-issues-warning-over-anti-flammatories.

6. WHO. Q: Could \#ibuprofen worsen disease for people with \#COVID19? 2020, March 19 [Available from: https://twitter.com/WHO/status/1240409217997189128.

7. Hoffmann M, Kleine-Weber H, Schroeder S, et al. SARS-CoV-2 Cell Entry Depends on ACE2 and TMPRSS2 and Is Blocked by a Clinically Proven Protease Inhibitor. Cell 2020 doi: 10.1016/j.cell.2020.02.052 [published Online First: 2020/03/07] 
8. Zhou P, Yang X-L, Wang X-G, et al. A pneumonia outbreak associated with a new coronavirus of probable bat origin. Nature2020;579(7798):270-73. doi: 10.1038/s41586-020-2012-7

9. Wan Y, Shang J, Graham R, et al. Receptor recognition by novel coronavirus from Wuhan: An analysis based on decade-long structural studies of SARS. Journal of Virology 2020:JVI.00127-20. doi: 10.1128/jvi.00127-20

10. Fang L, Karakiulakis G, Roth M. Are patients with hypertension and diabetes mellitus at increased risk for COVID-19 infection? The Lancet Respiratory Medicine 2020;8(4):e21. doi: 10.1016/S2213$2600(20) 30116-8$

11. Wan Y, Shang J, Graham R, et al. Receptor Recognition by the Novel Coronavirus from Wuhan: an Analysis Based on Decade-Long Structural Studies of SARS Coronavirus. Journal of Virology2020;94(7):e0012720. doi: $10.1128 / J V I .00127-20$

12. Li XC, Zhang J, Zhuo JL. The vasoprotective axes of the renin-angiotensin system: Physiological relevance and therapeutic implications in cardiovascular, hypertensive and kidney diseases.Pharmacol Res 2017;125(Pt A):21-38. doi: 10.1016/j.phrs.2017.06.005 [published Online First: 2017/06/18]

13. Li XC, Zhang J, Zhuo JL. The vasoprotective axes of the renin-angiotensin system: Physiological relevance and therapeutic implications in cardiovascular, hypertensive and kidney diseases.Pharmacol Res 2017;125(Pt A):21-38. doi: 10.1016/j.phrs.2017.06.005 [published Online First: 06/12]

14. Brown JD. Antihypertensive drugs and risk of COVID-19? The Lancet Respiratory Medicine doi: 10.1016/S2213-2600(20)30158-2

15. Fang L, Karakiulakis G, Roth M. Antihypertensive drugs and risk of COVID-19? \&\#x2013; Authors' reply. The Lancet Respiratory Medicine doi: 10.1016/S2213-2600(20)30159-4

16. Vaduganathan M, Vardeny O, Michel T, et al. Renin-Angiotensin-Aldosterone System Inhibitors in Patients with Covid-19. New England Journal of Medicine 2020 doi: 10.1056/NEJMsr2005760

17. Kelleni M. Drug induced malignancy: a focus on pioglitazone.Pharm Pharmacol Int $J$ 2018;6(4):297-99.

18. Qiao W, Wang C, Chen B, et al. Ibuprofen attenuates cardiac fibrosis in streptozotocin-induced diabetic rats. Cardiology2015;131(2):97-106. doi: 10.1159/000375362 [published Online First: 2015/04/22]

19. University of Basel. Ibuprofen and COVID-19 2020, March 16 [Available from: https://www.unibas.ch/en/News-Events/News/Uni-Research/Ibuprofen-and-COVID-19-Setting-therecord-straight.html.

20. Kelleni M. Ibuprofen potential addition to COVID-19 early management protocols: could it be superior to paracetamol and hydroxychloroquine? 2020, May 6 [Available from: https://journal.sketchingscience.org/users/318758/articles/448656-ibuprofen-potential-addition-to-covid19-early-management-protocols-could-it-be-superior-to-paracetamol-and-hydroxychloroquine.

21. Kuba K, Imai Y, Rao S, et al. A crucial role of angiotensin converting enzyme 2 (ACE2) in SARS coronavirus-induced lung injury. Nature medicine 2005;11(8):875-79. doi: 10.1038/nm1267

22. Oudit GY, Kassiri Z, Jiang C, et al. SARS-coronavirus modulation of myocardial ACE2 expression and inflammation in patients with SARS.European journal of clinical investigation 2009;39(7):618-25. doi: 10.1111/j.1365-2362.2009.02153.x

23. Koitka A, Cooper ME, Thomas MC, et al. Angiotensin converting enzyme 2 in the kidney. Clin Exp Pharmacol Physiol 2008;35(4):420-5. doi: 10.1111/j.1440-1681.2008.04889.x [published Online First: 2008/03/01]

24. Wong DW, Oudit GY, Reich H, et al. Loss of angiotensin-converting enzyme-2 (Ace2) accelerates diabetic kidney injury. Am J Pathol2007;171(2):438-51. doi: 10.2353/ajpath.2007.060977 [published Online 
First: 2007/06/30]

25. Wakahara S, Konoshita T, Mizuno S, et al. Synergistic Expression of Angiotensin-Converting Enzyme (ACE) and ACE2 in Human Renal Tissue and Confounding Effects of Hypertension on the ACE to ACE2 Ratio.Endocrinology 2007;148(5):2453-57. doi: 10.1210/en.2006-1287

26. American College of Cardiology. HFSA/ACC/AHA Statement Addresses Concerns Re: Using RAAS Antagonists in COVID-19 2020, March 17 [Available from: https://www.acc.org/latestin-cardiology/articles/2020/03/17/08/59/hfsa-acc-aha-statement-addresses-concerns-re-using-raasantagonists-in-covid-19.

27. European Medicines Agency. EMA advises continued use of medicines for hypertension, heart or kidney disease during COVID-19 pandemic. 2020, March 27

28. Bean D, Kraljevic Z, Searle T, et al. Treatment with ACE-inhibitors is associated with less severe disease with SARS-Covid-19 infection in a multi-site UK acute Hospital Trust. medRxiv2020:2020.04.07.20056788. doi: $10.1101 / 2020.04 .07 .20056788$ 